\title{
André Didierjean, La madeleine et le savant. Balade proustienne du côté de la psychologie cognitive
}

\section{Aron Verga}

\section{(2) OpenEdition}

1 Journals

\section{Edizione digitale}

URL: http://journals.openedition.org/studifrancesi/1440

DOI: $10.4000 /$ studifrancesi. 1440

ISSN: 2421-5856

\section{Editore}

Rosenberg \& Sellier

\section{Edizione cartacea}

Data di pubblicazione: 1 décembre 2015

Paginazione: 625

ISSN: 0039-2944

\section{Notizia bibliografica digitale}

Aron Verga, «André Didierjean, La madeleine et le savant. Balade proustienne du côté de la psychologie cognitive», Studi Francesi [Online], 177 (LIX | III) | 2015, online dal 01 décembre 2015, consultato il 06 janvier 2021. URL: http://journals.openedition.org/studifrancesi/1440 ; DOI: https://doi.org/10.4000/ studifrancesi. 1440

Questo documento è stato generato automaticamente il 6 janvier 2021.

\section{(c)}

Studi Francesi è distribuita con Licenza Creative Commons Attribuzione - Non commerciale - Non opere derivate 4.0 Internazionale. 


\title{
André Didierjean, La madeleine et le savant. Balade proustienne du côté de la psychologie cognitive
}

\author{
Aron Verga
}

\section{NOTIZIA}

ANDRÉ DIDIERJEAN, La madeleine et le savant. Balade proustienne du côté de la psychologie cognitive, Paris, Éditions du Seuil, 2015 («Science Ouverte»), pp. 155.

1 Professore di psicologia cognitiva all'Université de Franche-Comté, André Didierjean affronta la questione del funzionamento della memoria nei dieci capitoli che compongono il suo saggio («L'apprentissage»; «Perception et attention»; «La mémoire à court terme»; «La mémoire à long terme»; «Les faux souvenirs»; «Être expert dans un domaine»; «La cognition au fil de la vie»; «Lire les visages»; «Le sentiment d'être soi»; «Aux frontières de la psychologie cognitive»).

2 La trattazione è spesso accompagnata da esempi di esperimenti svolti in passato da scienziati al fine di valutare le capacità di apprendimento o le condizioni della memoria, per esempio il problema della scacchiera senza due angoli oppure il fenomeno noto come "effetto cocktail party". Nel primo caso, togliendo i due angoli neri, il paziente dovrebbe dire se è possibile ricoprire la scacchiera con le tessere del domino. Il secondo esperimento prevede di ricordare le parole dette in un orecchio e di ignorare quelle dette nell'altro. Al di là dei vari esperimenti e delle ricerche scientifiche presentate, la grande novità proposta da André Didierjean sta nell'affiancare ad essi dei brani di À la recherche du temps perdu di Marcel Proust. Si tratta pertanto di un'opera che getta le basi di un ponte tra una scienza considerata obiettiva e la scrittura romanzesca. Da una parte la psicologia cognitiva, che cerca di descrivere i meccanismi del funzionamento dei processi cognitivi (memoria, apprendimento, percezione ecc.). Dall'altra Marcel Proust, uno dei più grandi romanzieri del xx secolo, del quale si rivela 
la capacità di anticipare talvolta le conclusioni dei ricercatori contemporanei nelle sue analisi dei meccanismi cognitivi. Eccellendo nella descrizione dei diversi comportamenti dell'essere umano (quando impara, ragiona, si ricorda), le citazioni proustiane sono dunque utilizzate da André Didierjean per illustrare meglio le sue teorie scientifiche.

3 Scopriamo così che Proust evocava già nella Prisonnière la capacità dell'essere umano di apprendere le regole astratte di una lingua sconosciuta semplicemente entrandovi in contatto di frequente. Oppure, sempre nello stesso volume della Recherche, Proust aveva intuito che l'essere umano vive costantemente in un processo di apprendimento, ma non è in grado di memorizzare tutto. E proprio di ciò si rammarica il narratore durante un attacco di gelosia, poiché avrebbe voluto ricordare con precisione tutte le parole di Albertine.

4 Lungo tutto il saggio di André Didierjean la psicologia cognitiva si intreccia dunque alle pagine della Recherche, che in alcuni frangenti in maniera pionieristica precorrevano $\mathrm{i}$ tempi. «Cependant, heureusement pour la psychologie cognitive, ces moments sont rares» (p. 10). 